\title{
A triple active galactic nucleus in the NGC 7733-7734 merging group
}

\author{
Jyoti Yadav ${ }^{1,2} \odot$, Mousumi $\operatorname{Das}^{1} \oplus$, Sudhanshu Barway ${ }^{1} \odot$, and Francoise Combes $^{3} \odot$ \\ 1 Indian Institute of Astrophysics, Koramangala II Block, Bangalore 560034, India \\ e-mail: jyoti@iiap.res.in \\ 2 Pondicherry University, R.V. Nagar, Kalapet 605014, Puducherry, India \\ 3 Observatoire de Paris, LERMA, College de France, CNRS, PSL University, Sorbonne University, 75014 Paris, France
}

Received 29 April 2021 / Accepted 22 June 2021

\begin{abstract}
Context. Galaxy interactions and mergers can lead to supermassive black hole (SMBH) binaries, which become active galactic nucleus (AGN) pairs when the SMBHs start accreting mass. If there is a third galaxy involved in the interaction, then a triple-AGN system can form.

Aims. Our goal is to investigate the nature of the nuclear emission from the galaxies in the interacting pair NGC 7733-NGC 7734 using archival VLT/MUSE integral field spectrograph data and study its relation to the stellar mass distribution traced by near-infrared (NIR) observations from the South African Astronomical Observatory (SAAO).

Methods. We conducted NIR observations using the SAAO and identified the morphological properties of bulges in each galaxy. We used MUSE data to obtain a set of ionized emission lines from each galaxy and studied the ionization mechanism. We also examined the relation of the galaxy pair with any nearby companions with far-ultraviolet observations using the UVIT.

Results. The emission line analysis from the central regions of NGC 7733 and NGC 7734 shows Seyfert and low ionization nuclear emission-line regions type AGN activity. The galaxy pair NGC 7733-34 also shows evidence of a third component, which has Seyfertlike emission. Hence, the galaxy pair NGC 7733-34 forms a triple-AGN system. We also detected an extended narrow-line region associated with the nucleus of NGC 7733 .
\end{abstract}

Key words. galaxies: individual: NGC7733 - galaxies: individual: NGC7734 - galaxies: interactions - galaxies: active galaxies: Seyfert - techniques: imaging spectroscopy

\section{Introduction}

Galaxy interactions and mergers are the major drivers of galaxy evolution in our low redshift universe, leading to the growth of supermassive black holes (SMBHs), bulges, and massive galaxies (Di Matteo et al. 2005). One of the most favorable environments for such activity are galaxy groups where galaxies are closely interacting, especially those that have significant reservoirs of cold gas that can be used to fuel star formation and active galactic nuclear (AGN) activity (Eastman et al. 2007; Georgakakis et al. 2008; Arnold et al. 2009; Martini et al. 2009; Tzanavaris et al. 2014).

The tidal forces during galaxy interactions may trigger the formation of bars and spiral arms that produce gravitational torques on the stars and gas in the galaxy disks. These, in turn, drive gas into the nuclear regions, triggering central star formation (Springel et al. 2005; Hopkins \& Quataert 2011). The gas accretion onto the SMBHs can trigger AGN activity, but the details of how the gas reaches the inner-kiloparsec region are still not clear. However, processes such as nuclear spirals and kiloparsec-scale bars likely play an important role (Combes 2001). Observations suggest that most galaxies are associated with groups or have some companions (McGee et al. 2009). The galaxy interactions within the group can strongly affect the galaxy morphologies, and they can trigger star formation and AGN activity if the galaxies are gas rich (Duc \& Renaud 2013). Gas can also be pulled out due to tidal interaction and ram pressure stripping, as seen in the tails of $\mathrm{HI}$ gas or starforming knots in $\mathrm{H} \alpha$ and ultraviolet (UV; Kenney et al. 2004; von der Linden et al. 2010; Yadav et al. 2021).

As galaxies merge, the dynamical friction on the SMBHs in the individual galaxies causes them to move closer together. If the SMBHs are accreting, they can form AGN pairs (Rubinur et al. 2019). Studies show that there is a higher fraction of dual AGN in interacting galaxies, which indicates that galaxy interactions can trigger AGN activity (Satyapal et al. 2014; Kocevski et al. 2015; Koss et al. 2018). Several dual AGN have been detected locally (Koss et al. 2012; Rubinur et al. 2018; Imanishi et al. 2020) and at higher redshifts (Myers et al. 2008; Hennawi et al. 2010; Silverman et al. 2020; Silva et al. 2021). However, only a few binary AGN have been detected (Kharb et al. 2017).

Galaxy interactions can also lead to triple merger systems, and if the SMBHs of the individual galaxies are accreting, a triple-AGN system will form. Although such systems are rare, a few have been discovered (Koss et al. 2012; Pfeifle et al. 2019). It is important to detect more multiple AGN in order to understand how AGN activity can affect the merging processes on kiloparsec scales. In this Letter we present the detection of a kiloparsec-scale triple-AGN system in the nearby 
Table 1. Details of the galaxies.

\begin{tabular}{lccc}
\hline \hline Source & $\begin{array}{c}\text { RA } \\
\text { (hh mm ss) }\end{array}$ & $\begin{array}{c}\text { Dec } \\
\text { (dd mm ss) }\end{array}$ & $z$ \\
\hline NGC 7733 & $23: 42: 32.79$ & $-65: 57: 27.42$ & $0.03392 \pm 0.00003$ \\
NGC 7734 & $23: 42: 43.20$ & $-65: 56: 44.19$ & $0.03527 \pm 0.00006$ \\
NGC 7733N & $23: 42: 32.25$ & $-65: 57: 09.43$ & $0.03619 \pm 0.00012$ \\
2MASS 2342 & $23: 42: 49$ & $-65: 58: 26$ & \\
\hline
\end{tabular}

Notes. Columns 2 and 3 are the coordinates of galaxies in J2000.0. Column 4 is the redshift of NGC7733 and NGC7734 taken from Mathewson \& Ford (1996) and de Vaucouleurs et al. (1991), respectively.

galaxy pair NGC 7733-NGC 7734 (hereafter NGC 7733-34). The instruments, methods, and results are described below. The value of the Hubble constant $\left(H_{0}\right)$ used in this Letter is $67.8 \mathrm{~km} \mathrm{~s}^{-1} \mathrm{Mpc}^{-1}$

\section{NGC 7733-34 group}

NGC 7733-34 is a group of interacting galaxies at a distance of $154 \mathrm{Mpc}$ (Arp \& Madore 1987). NGC 7734 is at a distance of $48.3 \mathrm{kpc}$ and has a velocity of $406 \mathrm{~km} \mathrm{~s}^{-1}$ with respect to NGC 7733 (de Vaucouleurs et al. 1991). NGC 7733 is a barred spiral that shows knots in the arms and hosts a Seyfert 2 nucleus (Tempel et al. 2016). NGC 7734 is a barred spiral with peculiar arms (de Vaucouleurs et al. 1991; Paturel et al. 1989). The atomic gas content of the galaxy pair is $\leq 4.2 \times 10^{9} M_{\odot}$, and the molecular content of NGC 7733 and NGC 7734 is $\leq 15.4 \times$ $10^{8} M_{\odot}$ and $\simeq 1.073 \times 10^{10} M_{\odot}$, respectively (Horellou \& Booth 1997). Jahan-Miri \& Khosroshahi (2001) identified the starforming knots and their ages in the individual galaxies and found that NGC 7733 has a much younger stellar population than NGC 7734. The properties of the galaxies are given in Table 1.

\section{Observations and data analysis}

\subsection{UV data}

We observed NGC 7733-34 in the far-ultraviolet (FUV) band using the Ultraviolet Imaging Telescope (UVIT) on board ASTROSAT (Kumar et al. 2012). The UVIT has two telescopes that can simultaneously observe in three bands: FUV (1300-1800 ̊), near-ultraviolet (NUV; 2000-3000 $)$ ), and the visible band. Drift correction was done with the visible channel. The UVIT has a spatial resolution of around $1^{\prime \prime}$ and a field of view of $28^{\prime}$. We reduced the UVIT level 1 data downloaded from the Indian Space Science Data Centre (ISSDC) using CCDLAB (Postma \& Leahy 2017, 2020).

\subsection{Near-infrared data}

We performed deep near-infrared (NIR) imaging of the galaxy group NGC 7733-34 with the SIRIUS camera (Nagayama et al. 2003) on the Infrared Survey Facility (IRSF) $1.4 \mathrm{~m}$ telescope at South African Astronomical Observatory (SAAO) Sutherland, South Africa. The SIRIUS camera has three $1024 \times 1024$ $\mathrm{HgCdTe}$ detectors, which give simultaneous imaging in the $J$, $H$, and $K$ bands, with a field of view of $7.7^{\prime} \times 7.7^{\prime}$, and the pixel scale is $0.45^{\prime \prime}$ (Nagayama 2012). The images were taken in automatic dithering mode with $\sim 20^{\prime \prime}$ steps with individual frame exposure times of $30 \mathrm{~s}$ each, giving a total exposure time of typically $\sim 120 \mathrm{~min}$ for the combined set of exposures. Data reduction was carried out with a pipeline for the SIRIUS observations, including corrections for nonlinearity, dark subtraction, and flat fielding.

\subsection{MUSE data}

We used Multi Unit Spectroscopic Explorer (MUSE) archival data to study the optical emission lines from NGC 7733-34. MUSE is an integral field spectrograph (IFU) on the Very Large Telescope (VLT) and gives 3D spectroscopic data cubes with very high resolution. We used the data from the wide-field mode, which has a field of view of $1^{\prime} \times 1^{\prime}$. It has a spatial sampling of $0.2^{\prime \prime} \times 0.2^{\prime \prime}$ and a spectral resolution of 1750 at $4650 \AA$ to 3750 at $9300 \AA$.

We ran the Galaxy IFU Spectroscopy Tool $\left(\right.$ GIST $^{1}$ version 3.0.3; Bittner et al. 2019) pipeline to analyze the MUSE data. GIST uses a python implemented version of GANDALF (Sarzi et al. 2006; Falcón-Barroso et al. 2006; Bittner et al. 2019) for fitting the gas emission lines and the Penalized PixelFitting (pPXF; Cappellari \& Emsellem 2004; Cappellari 2017) method for stellar continuum fitting. GIST gives stellar kinematics and gas emission line properties based on binned data over a given wavelength range and signal-to-noise ratio $(\mathrm{S} / \mathrm{N})$. For stellar velocity calculations, the code convolves the linear combination of spectral energy templates with line of sight velocity distribution and fits that with the spectra from each bin.

We Voronoi binned the data based on $\mathrm{H} \alpha(6555-6575 \AA)$ emission. For binning, we used an $\mathrm{S} / \mathrm{N}$ of 30 . We also corrected the spectra for the Milky Way extinction. We adopted the method used in Comerón et al. (2021) and fitted the continuum using a multiplicative eighth-order Legendre polynomial. We used a minimum $\mathrm{S} / \mathrm{N}$ of 3 to remove noisy data before binning the data. We used GIST emission line fluxes for [O III], [N II], $\mathrm{H} \alpha$, and $\mathrm{H} \beta$ for further analysis.

Figure 1 shows the FUV, $\mathrm{H} \alpha$, and NIR $J$ band images of the galaxy group. The FUV and $J$ band images reveal an extended source (2MASS 23424898-6558257, referred to as 2MASS 2342) southeast of the galaxy NGC 7733. The FUV image reveals a bright bridge in the southeastern region connecting NGC 7733 to 2MASS 2342. The extended object in the southeastern region of NGC 7733 could be interacting with NGC 7733 and forms part of the NGC 7733-34 group. The projected spatial separation of 2MASS 2342 from NGC 7733 is $\sim 87 \mathrm{kpc}$, assuming that both galaxies are at the same redshift.

\section{Nature of the emission from the nuclei in NGC 7733-34}

To study the mechanism producing the ionized gas, we derived the Baldwin-Phillips-Terlevich (BPT; Baldwin et al. 1981) plots of the two galaxies. The BPT diagram uses the [O III] $\lambda 5007 / \mathrm{H} \beta$ and the $[\mathrm{N} \mathrm{II}] \lambda 6563 / \mathrm{H} \alpha$ line ratios to classify the origin of the emission. Figures $2 \mathrm{a}$ and $\mathrm{b}$ show the BPT plots of NGC 7733 and NGC 7734, respectively. The nuclear emission from NGC 7733 lies in the Seyfert part of the BPT plot, while NGC 7734 shows low ionization nuclear emission-line regions (LINER) emission from the central region. The BPT diagram of NGC 7733 also shows the ongoing star formation in the arms and Seyfert-like emission along the minor axis of the bar.

1 https://abittner.gitlab.io/thegistpipeline/ 

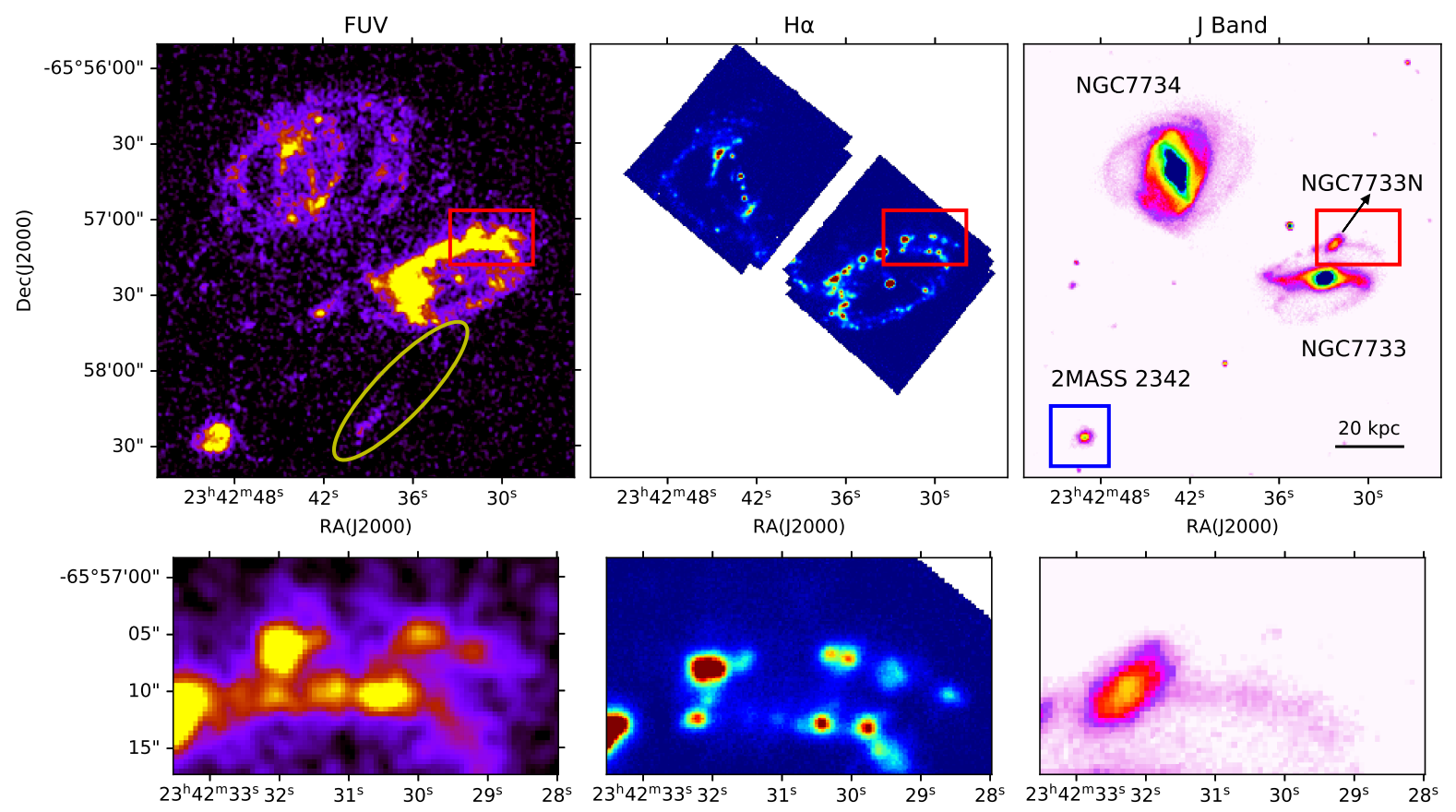

Fig. 1. Multiwavelength images of NGC 7733-7734 group. Upper panel: FUV, H $\alpha$, and $J$ band images of galaxy group NGC 7733-34. Bottom panel: zoomed-in version of the red rectangles. The blue box represents the possible merging galaxy 2MASS 2342, and the yellow ellipse shows the bridge between NGC 7733 and the merger candidate 2MASS 2342.

(a)

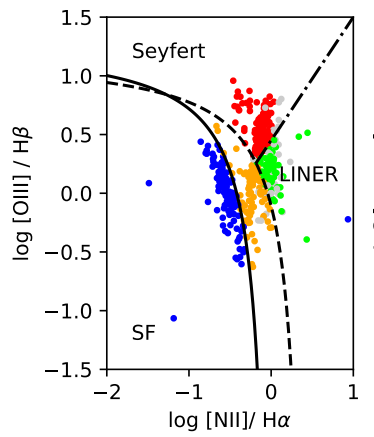

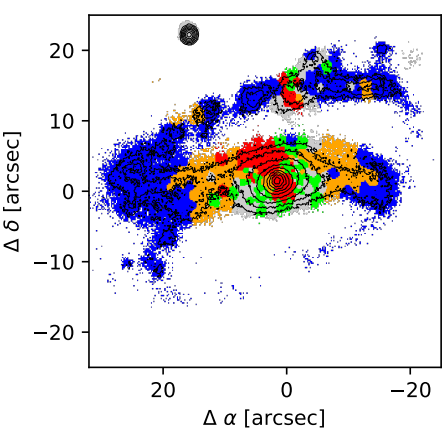

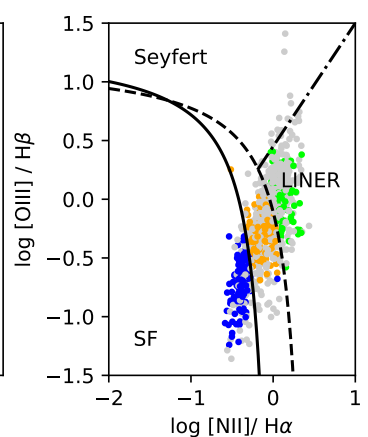

(b)

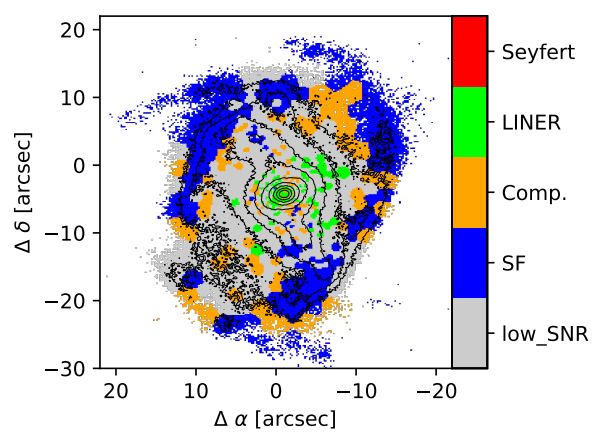

Fig. 2. [N II] BPT diagrams of (a) NGC 7733 and (b) NGC 7734. The $\Delta \alpha$ and $\Delta \delta$ are right ascension and declination with respect to the central position of the source, which is indicated in Table 1. The solid curve in the BPT diagrams is taken from Kauffmann et al. (2003), and the region below this line shows the ionization due to star-forming regions. The dashed curve is taken from Kewley et al. (2001), and the region above this line shows the ionization due to AGN. The dashed-dotted line is taken from Schawinski et al. (2007), which divides the LINER and Seyfert AGN. The bins with an amplitude-to-noise ratio $(\mathrm{A} / \mathrm{N})$ of less than 4 in any of the lines are shown in gray.

Figure 3 shows the color composite image of $\mathrm{H} \alpha$ (blue), [O III] (green), and [N II] (red) emissions, respectively. The bright $\mathrm{H} \alpha$ emission, in blue along the arms of NGC 7733, confirms the ongoing star formation in the arms of the galaxy. The [O III] emission is extended toward the northeast, which confirms that it is due to emission from the extended narrow-line region (ENLR) along the minor axis of the galaxy. The orientation is due to projection effects. The ENLR is associated with photoionized gas around the AGN and can be traced out to $\sim 18 \mathrm{kpc}$ in the northeastern region of NGC 7733. The velocity field and the trailing spirals suggest that the northeastern side is the near side of the galaxy, so the [O III] emission is extended toward us. The southeastern ENLR is extended away from our line of sight and is not visible due to obscuration by the dust in the galaxy. Ongoing star formation activity in NGC 7733 (Jahan-Miri \& Khosroshahi 2001) suggests the presence of gas and dust in this galaxy.

\section{Signatures of a third galaxy}

The FUV, $J$ band, and MUSE H $\alpha$ images show a large bright knot in the northern arm of NGC 7733. This region was identified as a star-forming knot in Jahan-Miri \& Khosroshahi (2001). The knot appears to be extended in the $J$ band, as shown in the red box in Fig. 1. Figure 4 shows the velocity field of the galaxy NGC 7733. Most of the velocity field shows features typical of a rotating disk, with the Doppler-shifted velocities of the approaching (blue) and receding sides (yellow and red). However, the velocity of the knot (shown in the rectangle) is clearly different from the velocity field of the disk.

\subsection{Decomposition using GALFIT}

We decomposed the NIR images of NGC 7733 and NGC 7734 into bulge, disk, and bar components using the GALFIT 


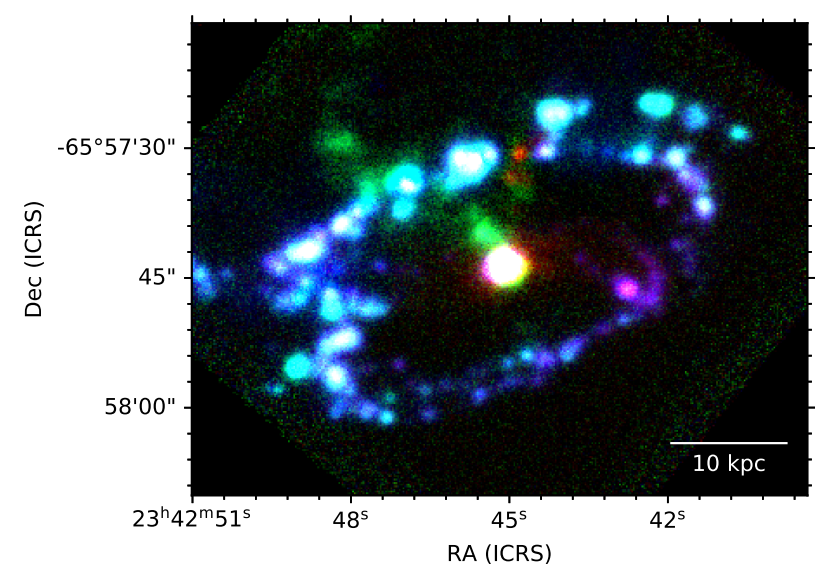

Fig. 3. Color composite image of NGC 7733. Blue, green, and red represent the $\mathrm{H} \alpha$, [O III] , and [N II] emissions, respectively. The [O III] emission toward the northeastern side shows the ENLR.

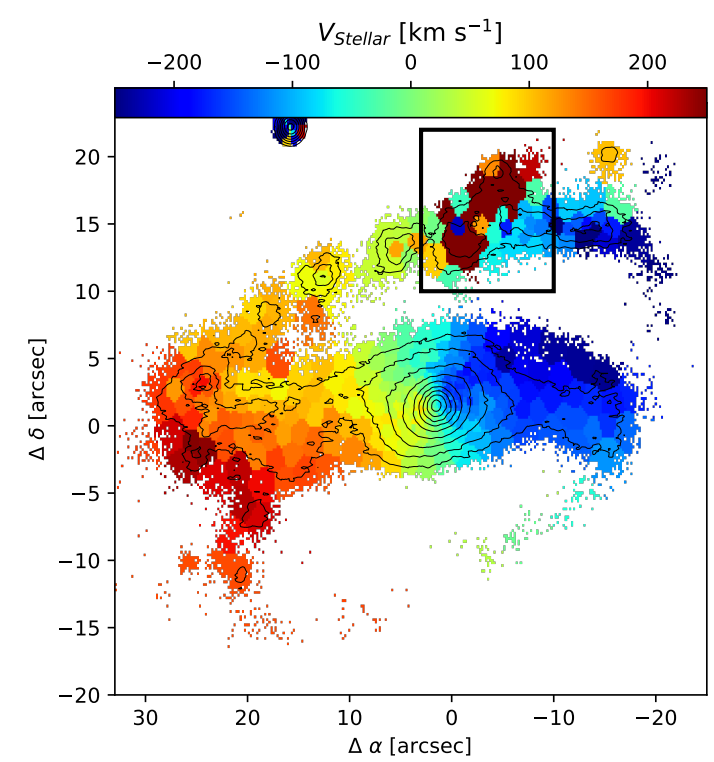

Fig. 4. Stellar velocity map of NGC 7733 obtained from GIST. The $\Delta \alpha$ and $\Delta \delta$ are right ascension and declination with respect to the central position of the source, which is indicated in Table 1 . The black rectangle in the northwest shows that the velocity of NGC $7733 \mathrm{~N}$ is different from that of galactic rotation.

(Peng et al. 2002) 2D decomposition code. We modeled both the bulges and the bars using Sersic profiles, and the disks with exponential profiles. A $\chi^{2}$ minimization technique was used to carry out this fit. We also used the Sersic profile to fit the knot on the northwest arm of NGC 7733 using GALFIT. We found that the effective radius, $r_{\mathrm{e}}$, of the Sersic profile in the $J$ band is $3.204^{\prime \prime}$, which corresponds to $\sim 2.4 \mathrm{kpc}$. Such a large value for the radius of the knot suggests that it is likely to be the bulge of a galaxy, instead of being a star-forming region within NGC 7733 .

\subsection{Emission line analysis of the knot in NGC 7733}

The knot shows a different velocity compared to the northern arm of NGC 7733, as indicated by the rectangle in Fig. 4. The MUSE spectra from this region show that there are two sets of emission lines, as shown in Fig. 5. The knot appears to be redshifted by $\sim 650 \mathrm{~km} \mathrm{~s}^{-1}$ with respect to the galaxy. This is a strong indication that the knot is not a part of the galaxy and

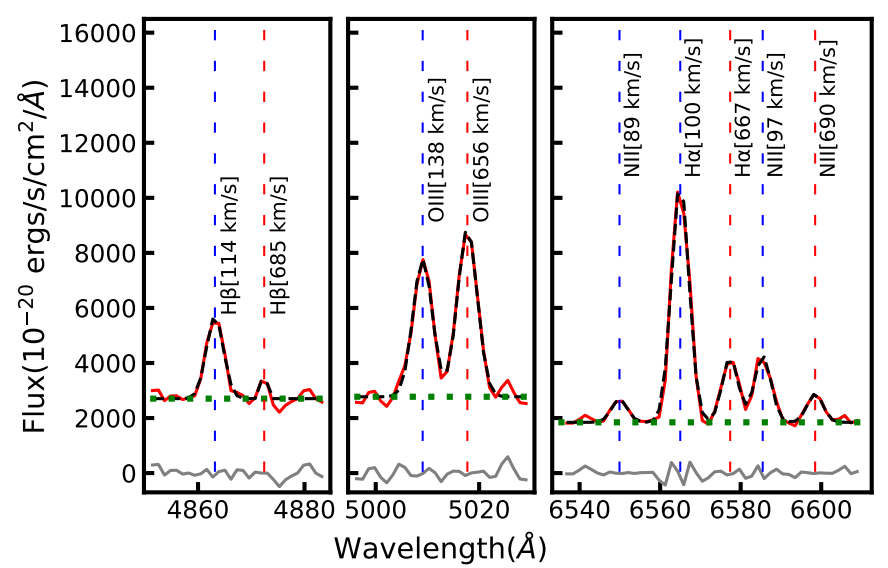

Fig. 5. Emission line fitting of NGC 7733 and NGC 7733N. The blue and red vertical dashed lines correspond to the spectra of NGC 7733 and NGC 7733N, respectively. Observed and fitted spectra are shown in red and black. The continuum is shown with dotted green horizontal lines. The vertical dashed lines show the peaks of emission lines along with their respective velocities.

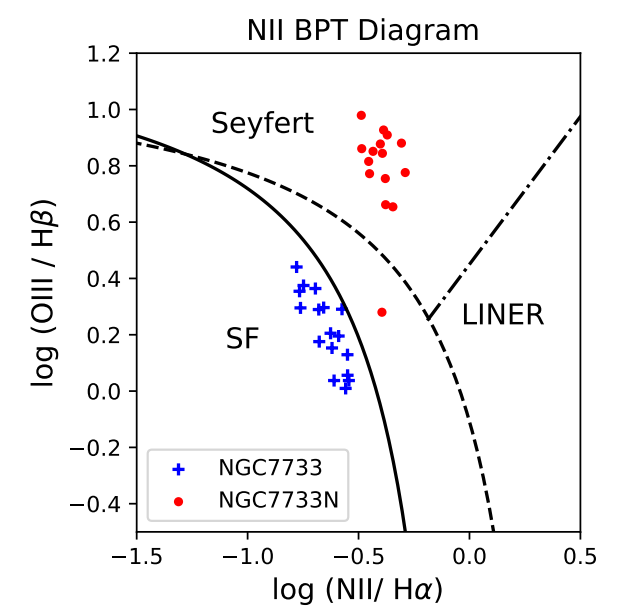

Fig. 6. BPT diagram for NGC 7733 and its companion NGC 7733N. Blue crosses and red dots represent the emission from NGC 7733 and NGC 7733N, respectively.

is instead a separate system that is visually overlapping with the arm of the galaxy. We use "NGC 7733N" to refer to the knot in the following text. To check the nature of emission from NGC 7733N, we used the fluxes of $\mathrm{H} \beta$, [O III], $\mathrm{H} \alpha$, and [N II] emission lines for all bins in the rectangle shown in Fig. 4. We divided the spectrum into three wavelength ranges, 4851-4883, 4996-5029, and 6532-6608 $\AA$. We fitted Gaussian profiles to $\mathrm{H} \beta$, [O III], $\mathrm{H} \alpha$, and [N II] lines and to a constant continuum, as shown in Fig. 5. The emission lines from some of the bins could not be fitted due to poor $\mathrm{S} / \mathrm{N}$. The resultant fits confirmed that NGC 7733N has its own set of emission lines. We calculated the mean velocity of $\mathrm{H} \alpha$ in NGC 7733 and NGC 7733N after fitting the emission lines. We found that NGC7733N is moving with a mean velocity of $+655 \pm 46 \mathrm{~km} \mathrm{~s}^{-1}$ with respect to NGC 7733 and is at a redshift of $0.03619 \pm 0.00012$.

The BPT diagram of two sets of emission lines is shown in Fig. 6. The red markers and blue crosses show the emission from NGC 7733N and NGC 7733, respectively. The emission from the NGC 7733 component of this region is due to the star formation, which is expected since it originates from the arm. The emission 
Table 2. Properties of galactic bulges and central black holes.

\begin{tabular}{|c|c|c|c|c|c|c|c|c|c|c|c|}
\hline \multirow[t]{3}{*}{ Galaxy } & \multirow{3}{*}{$\begin{array}{c}M_{K} \\
(\mathrm{mag})\end{array}$} & \multirow{3}{*}{$\begin{array}{c}\mathrm{Re} \\
(\operatorname{arcsec})\end{array}$} & \multirow[t]{3}{*}{$\log \left(M_{\star}\right)$} & \multicolumn{8}{|c|}{ Black hole mass $\left(10^{8} M_{\odot}\right)$} \\
\hline & & & & \multicolumn{2}{|c|}{$M_{\bullet}-L_{K}$} & \multicolumn{3}{|c|}{$M_{\bullet}-\sigma$} & \multicolumn{3}{|c|}{$M_{\bullet}-M_{\text {bulge }}$} \\
\hline & & & & MH2003 & KH2013 & Gu2009 & MM2013 & KH2013 & MH2003 & MM2013 & KH2013 \\
\hline NGC 7733 & -24.09 & 1.73 & 11.39 & $1.83_{-0.43}^{+0.62}$ & $4.68_{-1.03}^{+1.14}$ & $0.54_{-0.05}^{+0.05}$ & $0.64_{-0.03}^{+0.03}$ & $1.24_{-0.07}^{+0.06}$ & $1.31_{-0.31}^{+0.89}$ & $1.86_{-0.56}^{+0.73}$ & $3.04_{-0.73}^{+0.91}$ \\
\hline NGC 7734 & -23.74 & 1.58 & 11.81 & $1.28_{-0.26}^{+0.36}$ & $3.18_{-0.77}^{+0.90}$ & $1.13_{-0.18}^{+0.20}$ & $1.70_{-0.17}^{+0.18}$ & $2.63_{-0.26}^{+0.28}$ & $0.90_{-0.21}^{+0.60}$ & $1.25_{-0.41}^{+0.57}$ & $1.98_{-0.52}^{+0.67}$ \\
\hline NGC 7733N & -22.11 & 3.1 & 9.96 & $0.23_{-0.01}^{+0.01}$ & $0.51_{-0.17}^{+0.24}$ & & & & $0.15_{-0.04}^{+0.10}$ & $0.19_{-0.09}^{+0.14}$ & $0.26_{-0.09}^{+0.14}$ \\
\hline
\end{tabular}

Notes. Column 2: $K$ band absolute magnitude of bulges. Column 3: Effective radius of bulges. Column 4: Stellar mass estimated in $K$ band using $M / L=0.6$ (McGaugh \& Schombert 2014). Columns 5 and 6: Mass of central black holes using $M_{\bullet}$ and $K$ band luminosity. Columns 7-9: Mass of central black holes using the $M_{\bullet}-\sigma$ relation. Columns 10-12: Mass of central black holes using the $M_{\bullet}-$ Mass of bulge relation.

References. MH2003: Marconi \& Hunt (2003). KH2013: Kormendy \& Ho (2013). Gu2009: Gültekin et al. (2009). MM2013: McConnell \& Ma (2013).

from NGC 7733N lies in the Seyfert region, indicating an AGN in this galaxy.

\section{Triple interacting galaxies}

\subsection{Tidal features in UV and $H \alpha$}

Figure 1 shows an arm-like structure above the main arm in the northern part of NGC 7733 in the UVIT FUV and MUSE $\mathrm{H} \alpha$ images. The arm or tidal tail starts from the western side and merges with the location of NGC 7733N. It is likely to have formed via the tidal interaction between NGC 7733 and NGC 7733N. There are bright UV and $\mathrm{H} \alpha$ knots in the tidal arm, which suggests that there is ongoing massive star formation triggered by the interaction. NGC 7733N then represents the bulge of the merging companion galaxy (Fig. 1), which may have already lost most of its disk mass in the tidal interaction.

\subsection{Masses of the central black holes in NGC 7733, NGC 7733 N, and NGC 7734}

We estimated the $K$ band magnitude and effective radius of the bulges in NGC 7733, NGC 7733N, and NGC7734 using GALFIT and corrected the magnitude for Milky Way extinction using Schlafly \& Finkbeiner (2011). We estimated the stellar mass $\left(M_{\star}\right)$ of each galaxy using the mass-luminosity relation (McGaugh \& Schombert 2014). Based on the stellar mass ratios, NGC 7733-NGC 7734 is a major merging system and NGC 7733N-NGC 7733 is a minor merging system. The nuclear black hole masses $\left(M_{\bullet}\right)$ were estimated using the following relations: (i) $M_{\bullet}-$ luminosity in the $K$ band (Marconi \& Hunt 2003; Kormendy \& Ho 2013), (ii) the $M-\sigma$ relation (Gültekin et al. 2009; McConnell \& Ma 2013; Kormendy \& Ho 2013), and the (iii) $M_{\bullet}$-bulge mass relation (Marconi \& Hunt 2003; McConnell \& Ma 2013; Kormendy \& Ho 2013). The SMBH masses for the three AGN and the stellar masses of the galaxies are listed in Table 2. The bulge velocity dispersion of NGC 7733N is not reliable, so we could not calculate the black hole mass in NGC $7733 \mathrm{~N}$ using the $M-\sigma$ relation. The nuclear black hole masses of NGC $7733\left(0.54-4.68 \times 10^{8} M_{\odot}\right)$ and NGC $7734\left(0.90-3.18 \times 10^{8} M_{\odot}\right)$ have a similar range of values, and their stellar masses are also comparable. NGC 7733N hosts the least massive stellar disk and the least massive nuclear black hole $\left(1.53-5.15 \times 10^{7} M_{\odot}\right)$ in the triple-AGN system.

\section{Implications for our triple-AGN detection}

Dual AGN have been detected in many galaxies, but triple AGN are rare. Our study shows that multiple-AGN systems can be present in galaxy groups that show ongoing mergers. Since galaxy groups are fairly common (Tempel et al. 2017), they can be good targets for detecting multiple AGN. Another important implication of our study is the enhanced AGN feedback in such systems, which can heat the intracluster medium in clusters and enrich the circumgalactic medium around galaxies (Fabian 2012; Tumlinson et al. 2017). Although multiple-AGN feedback is relatively unexplored, it must play an important role in building hot gas reservoirs around galaxies, especially at early epochs when galaxy mergers were more frequent (Lackner et al. 2014; Man et al. 2016; Mundy et al. 2017). Feedback processes such as jets or intense quasar outflows from AGN can affect the evolution of the host galaxy (Salomé et al. 2016; Harrison et al. 2018). Enhanced multiple-AGN feedback effects may lead to faster SMBH growth (Volonteri et al. 2020). There may be additional star formation if the AGN outflows interact with one another. Triple-AGN systems such as the one we have detected in NGC 7733-34 are thus ideal laboratories for studying the growth of hot gas, AGN feedback, and SMBH growth in mergers and should be relatively common in gas-rich galaxy groups.

\section{Conclusions}

Our main conclusions are the following.

1. We find that the paired galaxies NGC 7733 and NGC 7734 host LINER- and Seyfert-type nuclei, respectively.

2. We detected an ENLR from the AGN in NGC 7733 toward the northeastern region.

3. The multiwavelength data show a tidal arm above the northern arm of NGC 7733, and the tidal arm connects with the location of a third galaxy, which we call NGC 7733N.

4. We have confirmed the existence of the third galaxy, NGC 7733N, in the NGC 7733-34 group. It appears to overlap with the northern arm of NGC 7733. The emission line analysis of NGC 7733N shows that it hosts a Seyfert nucleus.

5. The UV and NIR images show a fourth galaxy (2MASS 2342) lying southeast of the galaxy pair, which could be part of the merging system. We see a potential bridge between NGC 7733 and 2MASS 2342 in the UV map. This bridge is composed of hot gas that had been pulled out during the interaction phase. However, redshift estimation is necessary to confirm this. 
Triple AGN are rare; however, detailed studies could enhance their observed frequency in groups, and especially at high redshift. Although this study focuses only on one system, the results suggest that small merging groups are ideal laboratories for studying multiple-AGN systems.

Acknowledgements. We thank the anonymous referee for the thoughtful review, which improved the impact and clarity of this work. This publication uses the data from the UVIT, which is part of the AstroSat mission of the Indian Space Research Organisation (ISRO), archived at the Indian Space Science Data Centre (ISSDC). This research has also used NIR data from IRSF at the South African Astronomical Observatory (SAAO). Part of the results are based on observations collected at the European Southern Observatory under ESO programme 0103.A-0637, run B. This research has made use of the NASA/IPAC Extragalactic Database (NED) which is operated by the Jet Propulsion Laboratory, California Institute of Technology, under contract with the National Aeronautics and Space Administration. M.D. acknowledges the support of Science and Engineering Research Board (SERB) MATRICS grant MTR/2020/000266 for this research.

\section{References}

Arnold, T. J., Martini, P., Mulchaey, J. S., Berti, A., \& Jeltema, T. E. 2009, ApJ, 707, 1691

Arp, H. C., \& Madore, B. 1987, A Catalogue of Southern Peculiar Galaxies and Associations (Cambridge: Cambridge University Press)

Baldwin, J. A., Phillips, M. M., \& Terlevich, R. 1981, PASP, 93, 5

Bittner, A., Falcón-Barroso, J., Nedelchev, B., et al. 2019, A\&A, 628, A117

Cappellari, M. 2017, MNRAS, 466, 798

Cappellari, M., \& Emsellem, E. 2004, PASP, 116, 138

Combes, F. 2001, in Advanced Lectures on the Starburst-AGN, eds. I. Aretxaga,

D. Kunth, \& R. Mújica, 223

Comerón, S., Knapen, J. H., Ramos Almeida, C., \& Watkins, A. E. 2021, A\&A, 645, A130

de Vaucouleurs, G., de Vaucouleurs, A., \& Corwin, H. G., Jr. 1991, Third Reference Catalogue of Bright Galaxies

Di Matteo, T., Springel, V., \& Hernquist, L. 2005, Nature, 433, 604

Duc, P. A., \& Renaud, F. 2013, in Tides in Colliding Galaxies, eds. J. Souchay, S. Mathis, \& T. Tokieda, 861, 327

Eastman, J., Martini, P., Sivakoff, G., et al. 2007, ApJ, 664, L9

Fabian, A. C. 2012, ARA\&A, 50, 455

Falcón-Barroso, J., Bacon, R., Bureau, M., et al. 2006, MNRAS, 369, 529

Georgakakis, A., Gerke, B. F., Nandra, K., et al. 2008, MNRAS, 391, 183

Gültekin, K., Richstone, D. O., Gebhardt, K., et al. 2009, ApJ, 698, 198

Harrison, C. M., Costa, T., Tadhunter, C. N., et al. 2018, Nat. Astron., 2, 198

Hennawi, J. F., Myers, A. D., Shen, Y., et al. 2010, ApJ, 719, 1672

Hopkins, P. F., \& Quataert, E. 2011, MNRAS, 415, 1027

Horellou, C., \& Booth, R. 1997, A\&AS, 126, 3

Imanishi, M., Kawamuro, T., Kikuta, S., Nakano, S., \& Saito, Y. 2020, ApJ, 891, 140
Jahan-Miri, M., \& Khosroshahi, H. G. 2001, AJ, 122, 792

Kauffmann, G., Heckman, T. M., Tremonti, C., et al. 2003, MNRAS, 346, 1055 Kenney, J. D. P., van Gorkom, J. H., \& Vollmer, B. 2004, AJ, 127, 3361

Kewley, L. J., Dopita, M. A., Sutherland, R. S., Heisler, C. A., \& Trevena, J. 2001, ApJ, 556, 121

Kharb, P., Lal, D. V., \& Merritt, D. 2017, Nat. Astron., 1, 727

Kocevski, D. D., Brightman, M., Koekemoer, A. M., \& Nandra, K. 2015, Are Compton-Thick AGN the Missing Link Between Mergers and Black Hole Growth?, HST Proposal

Kormendy, J., \& Ho, L. C. 2013, ARA\&A, 51, 511

Koss, M., Mushotzky, R., Treister, E., et al. 2012, ApJ, 746, L22

Koss, M. J., Blecha, L., Bernhard, P., et al. 2018, Nature, 563, 214

Kumar, A., Ghosh, S. K., Hutchings, J., et al. 2012, in Space Telescopes and Instrumentation 2012: Ultraviolet to Gamma Ray, eds. T. Takahashi, S. S. Murray, \& J. W. A. den Herder, SPIE Conf. Ser., 8443, 84431 N

Lackner, C. N., Silverman, J. D., Salvato, M., et al. 2014, AJ, 148, 137

Man, A. W. S., Zirm, A. W., \& Toft, S. 2016, ApJ, 830, 89

Marconi, A., \& Hunt, L. K. 2003, ApJ, 589, L21

Martini, P., Sivakoff, G. R., \& Mulchaey, J. S. 2009, ApJ, 701, 66

Mathewson, D. S., \& Ford, V. L. 1996, ApJS, 107, 97

McConnell, N. J., \& Ma, C.-P. 2013, ApJ, 764, 184

McGaugh, S. S., \& Schombert, J. M. 2014, AJ, 148, 77

McGee, S. L., Balogh, M. L., Bower, R. G., Font, A. S., \& McCarthy, I. G. 2009, MNRAS, 400, 937

Mundy, C. J., Conselice, C. J., Duncan, K. J., et al. 2017, MNRAS, 470, 3507

Myers, A. D., Richards, G. T., Brunner, R. J., et al. 2008, ApJ, 678, 635

Nagayama, T. 2012, African Skies, 16, 98

Nagayama, T., Nagashima, C., Nakajima, Y., et al. 2003, in Instrument Design and Performance for Optical/Infrared Ground-based Telescopes, eds. M. Iye, \& A. F. M. Moorwood, SPIE Conf. Ser., 4841, 459

Paturel, G., Fouque, P., Bottinelli, L., \& Gouguenheim, L. 1989, A\&AS, 80, 299

Peng, C. Y., Ho, L. C., Impey, C. D., \& Rix, H.-W. 2002, AJ, 124, 266

Pfeifle, R. W., Satyapal, S., Manzano-King, C., et al. 2019, ApJ, 883, 167

Postma, J. E., \& Leahy, D. 2017, PASP, 129, 115002

Postma, J. E., \& Leahy, D. 2020, PASP, 132, 054503

Rubinur, K., Das, M., \& Kharb, P. 2018, JApA, 39, 8

Rubinur, K., Das, M., \& Kharb, P. 2019, MNRAS, 484, 4933

Salomé, Q., Salomé, P., Combes, F., \& Hamer, S. 2016, A\&A, 595, A65

Sarzi, M., Falcón-Barroso, J., Davies, R. L., et al. 2006, MNRAS, 366, 1151

Satyapal, S., Ellison, S. L., McAlpine, W., et al. 2014, MNRAS, 441, 1297

Schawinski, K., Thomas, D., Sarzi, M., et al. 2007, MNRAS, 382, 1415

Schlafly, E. F., \& Finkbeiner, D. P. 2011, ApJ, 737, 103

Silva, A., Marchesini, D., Silverman, J. D., et al. 2021, ApJ, 909, 124

Silverman, J. D., Tang, S., Lee, K.-G., et al. 2020, ApJ, 899, 154

Springel, V., Di Matteo, T., \& Hernquist, L. 2005, ApJ, 620, L79

Tempel, E., Kipper, R., Tamm, A., et al. 2016, A\&A, 588, A14

Tempel, E., Tuvikene, T., Kipper, R., \& Libeskind, N. I. 2017, A\&A, 602, A100

Tumlinson, J., Peeples, M. S., \& Werk, J. K. 2017, ARA\&A, 55, 389

Tzanavaris, P., Gallagher, S. C., Hornschemeier, A. E., et al. 2014, ApJS, 212, 9

Volonteri, M., Pfister, H., Beckmann, R. S., et al. 2020, MNRAS, 498, 2219

von der Linden, A., Wild, V., Kauffmann, G., White, S. D. M., \& Weinmann, S. 2010, MNRAS, 404, 1231

Yadav, J., Das, M., Patra, N. N., et al. 2021, ApJ, 914, 54 